\title{
Analysis and optimization of rubidium spectrum lamp to eliminate frequency fluctuations of rubidium atomic frequency standard
}

\author{
Jiqing Lian ${ }^{1,2, a}$, Jinhai Zhang ${ }^{1}$, Fucheng Shi ${ }^{1}$, Jun Zhang ${ }^{1}$, Lixun Wei ${ }^{1}$ and Kuan Wang ${ }^{1}$ \\ ${ }^{1}$ Lanzhou Institute of Physics, 730000 Lanzhou, China \\ ${ }^{2}$ Science and Technology on Vacuum Technology and Physics Laboratory, 730000 Lanzhou, China
}

\begin{abstract}
Rubidium atomic frequency standard (RAFS) is the most widely used frequency standard in space. The light used to pump the atoms and detect the resonance signal is emitted by rubidium spectrum lamp, so the light intensity of rubidium spectrum lamp directly determines the performance of RAFS. This paper discussed on-board RAFS' output frequency fluctuations caused by rubidium spectrum lamp. The reason of frequency fluctuations from rubidium lamps was described. To obtain stable lamp light intensity, analysis and optimization of the lamp was developed. Relevant experiments were carried out to verify the optimization. The study content of this paper is beneficial to improve the performance of a single temperature controlled space RAFS physics package.
\end{abstract}

\section{Introduction}

Rubidium atomic frequency standard (RAFS) is the most widely used atomic clock in space, which is characterized by small size, low power consumption and good stability. RAFS has been successfully applied to the satellites for Navigation, remote sensing, reconnaissance and other applications. The accuracy and stability of RAFS is closely related to the performance of the satellite. For example, in the electronic system of a navigation technology satellite (NTS), the on-board RAFS, as the clock signal reference of the satellite, provides accurate and stable clock signals for the satellite electronic system, and its stability index directly affects the precision of the navigation system. Unfortunately, research shows that, when the satellites operate in space, especially in the first one or two years, frequency fluctuations may happen to the rubidium atomic frequency standard, which have bad effect on the accuracy and stability of the RAFS. Although the frequency fluctuations mainly decreased with the passage of time, they were still one of the main factors affecting the performance of space RAFS. Therefore, it is of great significance to reduce the frequency fluctuations of space RAFS.

\section{Description and analysis of frequency fluctuations}

According to reference ${ }^{[1][2]}$, most of the RAFSs on GPS IIR satellites had a general performance that is among the best of the constellation. However, there were still several RAFSs with worse performance mainly caused by frequency fluctuations. There were also frequency fluctuations in BDS satellite $\operatorname{RAFS}^{[3]}$. The fluctuations were mainly manifested in two ways: 1 , the fluctuations occurred periodically with the period about a few days; 2 , the frequency fluctuations occurred in the form of steps or jumps, but the magnitude of the frequency fluctuations tend to decay with time. The frequency fluctuations of the RAFSs on GPS IIR satellites were mainly manifested as the second kind, which were consistent with the ground test results. And our study is also mainly focused on the frequency jumps.

Space RAFS is subject to several anomalies that can be caused by the environment or internal factors. And frequency jumps are a common type of clock anomaly which directly impacts the performance of a satellite system, such as global navigation satellite system(GNSS) ${ }^{[4]}$. For the frequency jumps, the internal factors are more important. The study ${ }^{[5-7]}$ shows that frequency jumps and drift of RAFS are usually respectively accompanied by abnormal and slow light intensity changes (as shown in figure 1), and the researchers believe that the instability of rubidium spectral light intensity is one of the most important reasons for frequency jumps. A RAFS is composed of a circuit system and a physics package. Apparently, the reasons of the lamp intensity jumps include the noise of the circuit system (such as fluctuations of DC-DC's output) and the imperfect manufactured physics package. However, by monitoring the parameters of the on-board circuits, we can mainly rule out the influence of the circuit system.

\footnotetext{
${ }^{\mathrm{a}}$ Corresponding author: lianjiqing@ outlook.com
} 


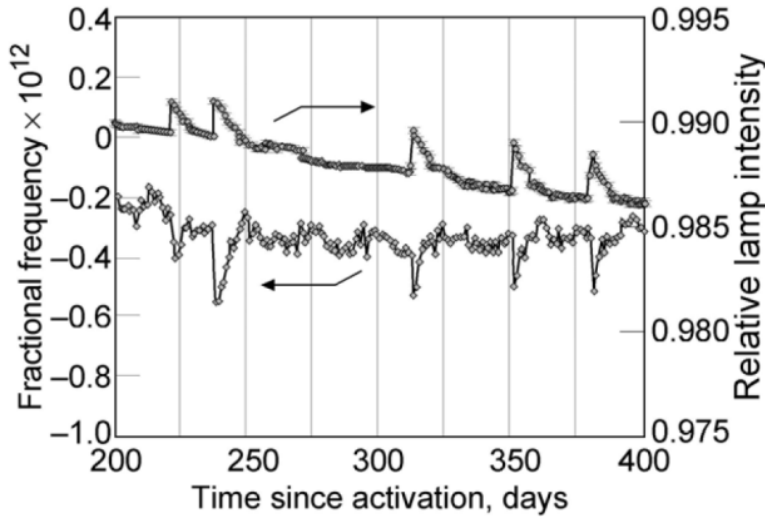

Figure 1. Jumps in the lamp intensity that coincide with jumps in the RAFS' frequency.

The physics package of a RAFS, as the block diagram shown in figure 2, contains three rubidium cells: a pure $\mathrm{Rb}-87$ lamp, a discrete $\mathrm{Rb}-85$ isotopic filter cell, and an $\mathrm{Rb}-87$ absorption cell. The cells are usually made of alkali-free glass. When the RAFS operates, all the cells work at high temperature. In particular, the lamp's working temperature usually reaches over $100^{\circ} \mathrm{C}$, which is dozens of degrees hotter than the other two cells'. The rubidium atoms in the cells will gradually decrease by permeating through the glass and chemical reaction at high temperature. In order to ensure that an on-board RAFS can operate for more than 10 years, the manufactures have to fill enough rubidium atoms to the cells ${ }^{[8]}$. The saturated vapor pressure of rubidium at a certain temperature is an invariant constant, so the excess rubidium atoms will adheres to the inner wall of the cells. There is anecdotal evidence that the frequency steps or jumps are related to excess rubidium.

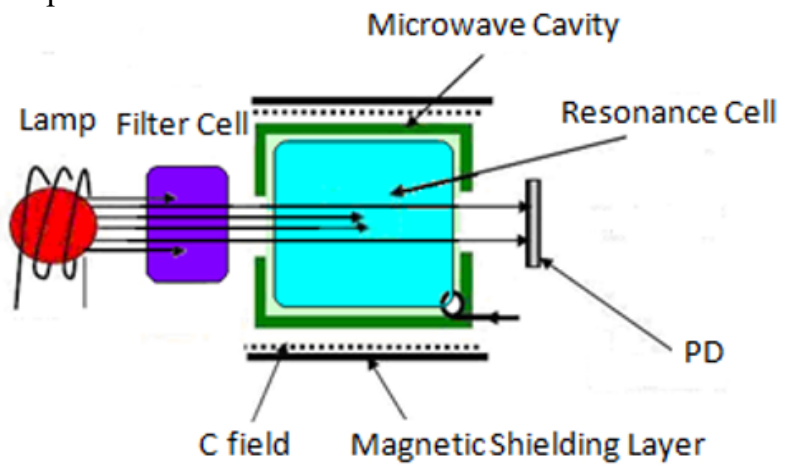

Figure 2. Schematic diagram of the physics package of RAFS.

For a RAFS, since the spectrum lamp works at a higher temperature, which leads to the faster rubidium consumption, rubidium atoms in the lamp should be more than that in the other two cells. So we believe that the excess rubidium in the lamp is the key factor of frequency jumps, and that is easily proved by testing a RAFS physics package with different lamps. A complete rubidium spectrum lamp module consists of a bulb, a exciter circuit and other structural components. Rubidium lamp exciter circuits supply about 0.5 watt of RF power at around $100 \mathrm{MHz}$ to lamp bulbs. The $\mathrm{Rb}$ lamp is usually excited inside a series-tuned coil connected to the circuit board, which is working as a microwave inductor of the LC oscillation network. Rubidium spectrum lamp is a kind of electrodeless discharge lamp. Under the RF electric field, the discharge gas molecules (such as
$\mathrm{Ar} / \mathrm{Kr} / \mathrm{Xe}$ ) in the lamp bulb ionize, and then the ions transfer energy to the rubidium atoms in gas state by collisions. The rubidium atoms in excited state will decay to the ground state by emitting photons. It is clear that the RF power is directly related to the lamp brightness, and the optimization of the RF power control was reported ${ }^{[9]}$, so it is not discussed in this paper.

When a rubidium spectrum lamp works in normal state, the excess rubidium atoms will be in liquid state. Usually, we make a "tail" in the end of a bulb(as shown in figure 3) to set a cold end for the accumulation of liquid rubidium. Obviously, it will take some time for the rubidium atoms in liquid state to achieve a stable distribution in the bulb. During the process of rubidium atoms' redistribution, the moving of the atoms may lead to the change of light intensity which will lead to the change of frequency. For different RAFSs, the processes of rubidium atoms' redistribution in the lamp bulbs last for different lengths of time. But overall, the distribution of rubidium in lamps tends to be more and more stable with the RAFSs' operating time. This is also consistent with the reduction of frequency jumps over operating time.

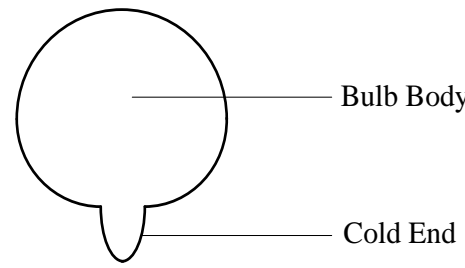

Figure 3. An typical diagram of lamp bulb.

\section{Analysis and optimization of rubidium spectrum lamps}

In order to abtain long-term and accurate clock signal for satellites' electric systems, frequency jumps of on-board RAFSs should be minimized or avoided. Through the analysis in the previous section, when the physics package is only considered, the frequency jumps of an on-board RAFS are mainly caused by the liquid rubidium atoms moving in the lamps to achieve the stable distribution. Rubidium atoms' stable distribution is closely related to the temperature field of lamp bulb, and the fluidity of liquid makes it difficult to hold the excess rubidium atoms in the optimal place just by optimize the manufacturing process of the lamp bulbs. Therefore, proper mechanical, electrical and thermal design of a rubidium spectrum lamp is very important, that will make the rubidium bulb obtain a temperature field more suitable and stable for liquid atoms to redistribute rapidly and smoothly.

\subsection{Original design and analysis of Rb spectrum lamp}

Figure 4 is the structure diagram of the rubidium spectrum lamp originally designed by Lanzhou Institute of Physics (LIP). From the bottom to the top, an aluminum baseplate, a RF excitation circuit board, an heat insulation structure, a cold end support structure, an 
series-tuned coil for excitation and a rubidium bulb are successively arranged. The whole lamp module is installed in an aluminium lamp room. When the RAFS operates, the RF energy is coupled to the rubidium bulb through the coil, and the temperature of the rubidium bulb is around $110^{\circ} \mathrm{C}$, and there must be a temperature gradient field over the wall of the bulb because of the cold end. When the rubidium bulb reaches thermal balance, the liquid rubidium would be stable kept in the cold end. Apparently, the lamp has no specialized heating circuit, all the heat of the bulb comes from the excitation circuit by RF power coupling and heat conduction. So we added a heat insulation structure to avoid excessive heat dissipation of the bulb.

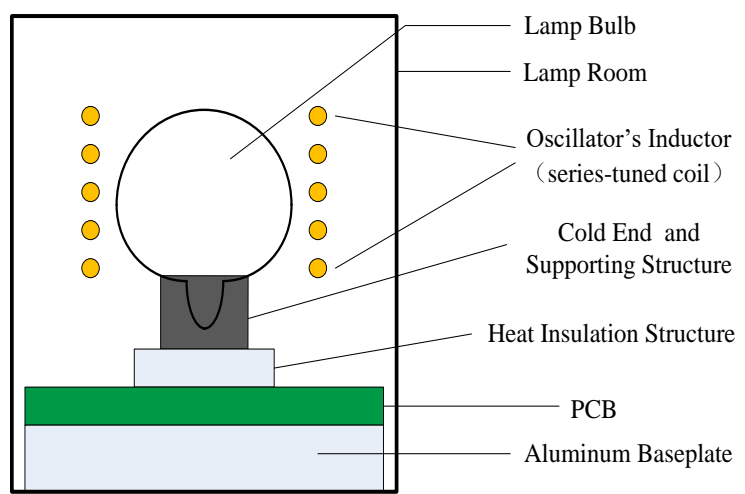

Figure 4. Structure diagram of the rubidium spectrum lamp originally designed.

Thermal simulation of the rubidium spectrum lamp was carried out by using ANSYS. The simulation result of the rubidium bulb is as shown in figure 5. The highest temperature of the bulb was about $117.53^{\circ} \mathrm{C}$, and the lowest temperature was about $113.07^{\circ} \mathrm{C}$. The rubidium bulb could form stable temperature gradient field, it means that the excess liquid rubidium atoms would finally flow to the cold end. However, the temperature gradient of the bulb was not steep enough, which limits the rate and stability of rubidium atoms redistribution. Besides, we can see that, most of the bulb body was at a high temperature. That might lead to the random moving of liquid atoms on the bulb body during the redistribution process, and result in frequency jumps of the RAFS, which is surely not desired.
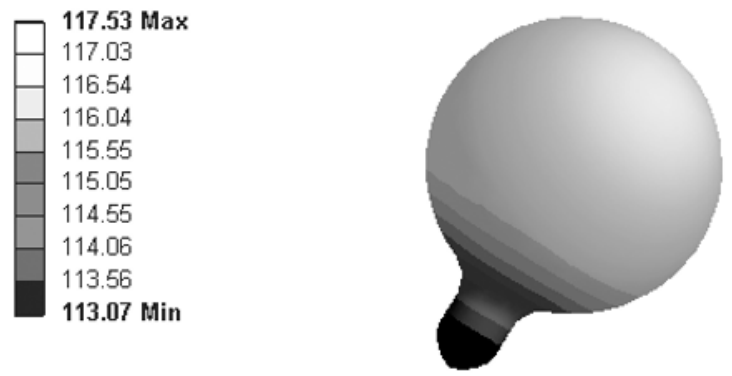

Figure 5. Thermal simulation result of the rubidium bulb.

\subsection{Optimization of Rb spectrum lamp}

In order to make rubidium distribute more rapidly and smoothly, better temperature gradient field must be obtained, and the time for thermal balance should be also shortened. We redesigned the lamp as shown in figure 6.

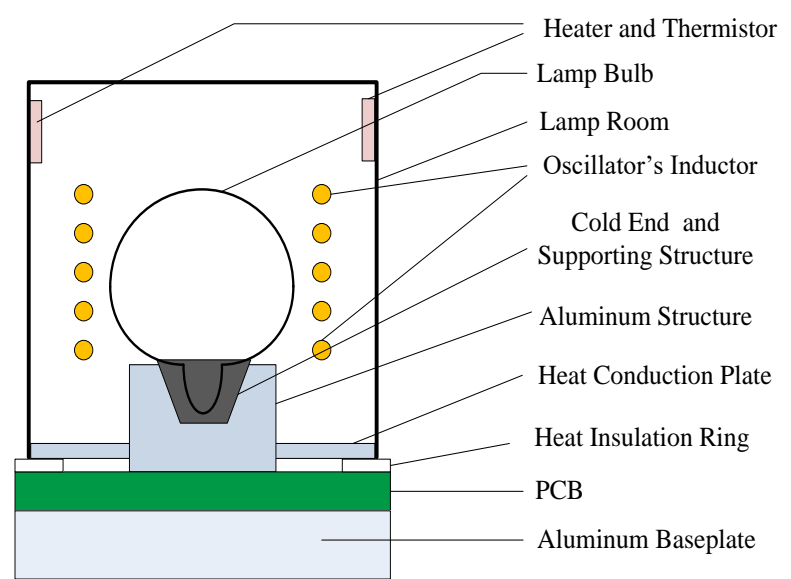

Figure 6. Optimized structure diagram of the rubidium spectrum lamp.

The optimization of rubidium spectrum lamp is mainly about the following aspects:

1)In the original design, all the heat of the bulb came from the excitation circuit, because we believe that single energy source has relatively lower noise and is better for more stable thermal balance and light intensity. However, that will take relatively more time for the whole lamp room to achieve stable thermal state and is not good for the lamp to obtain thermal balance rapidly. Aiming at this problem, we added a temperature control module(the heater and thermistor were installed in the specialized installation channels on the lamp room, while the control circuit was in a separate box for installing circuit system) to the lamp room to keep the aluminium lamp room at around $100^{\circ} \mathrm{C}$. According to the simulation and test result of the original lamp, the bulb in the coil works at a temperature over $110^{\circ} \mathrm{C}$, which is higher than the temperature of the lamp room we designed, so the added heating module would not influence the stable temperature of the bulb too much. The external constant temperature field applied to the lamp room would be conducive to rapid thermal balance of the spectrum lamp by transfer heat to the lamp through a heat conduction plate, and that would also be benificial to the quickstarting process of the lamp ${ }^{[10]}$.

2)A better temperature gradient field needs a relatively colder end. So we removed the heat insulation structure shown in figure 4, and added an aluminium structure to help heat conduction of the cold end. The aluminium structure was connected to the lamp room through the heat conduction plate mentioned in the previous paragraph.

3)In the original design, the excitation circuit board was inside of the room to provide heat to the whole lamp. With the specialized heating module added, it is not necessary for the lamp to get too much heat from the circuit board. On the contrary, working under a high temperature for long time would have a negative effect on the excitation circuit $^{[11]}$. So the lamp room was redesigned so that it was directly in contact with the heat conduction plate, and kept the excitation circuit board outside of the lamp room. Besides, a heat insulation ring 
with low thermal conductivity was added between the lamp room and the circuit board to avoid heat conduction.

Thermal simulation analysis of the optimized rubidium spectrum lamp module was developed using ANSYS, and the temperature gradient field of the rubidium bulb under stable operation is obtained in figure 7. During the stable operation of rubidium spectrum lamp, the highest temperature of rubidium bulb was about $120.26^{\circ} \mathrm{C}$, and the lowest temperature was about $100.06^{\circ} \mathrm{C}$. Compared with the original design, a better temperature gradient field was formed in the whole rubidium bulb (including the main body of the rubidium bulb) and the cold end had lower temperature. That is consistent with our design purpose, and beneficial for the liquid rubidium atoms to flow to the cold end rapidly and could also make the distribution of liquid rubidium more stable. According to the previous analysis of frequency jumps of RAFS, we believe that this optimization of rubidium spectrum lamp can effectively reduce the frequency jumps caused by the unstable rubidium distribution in the lamp.

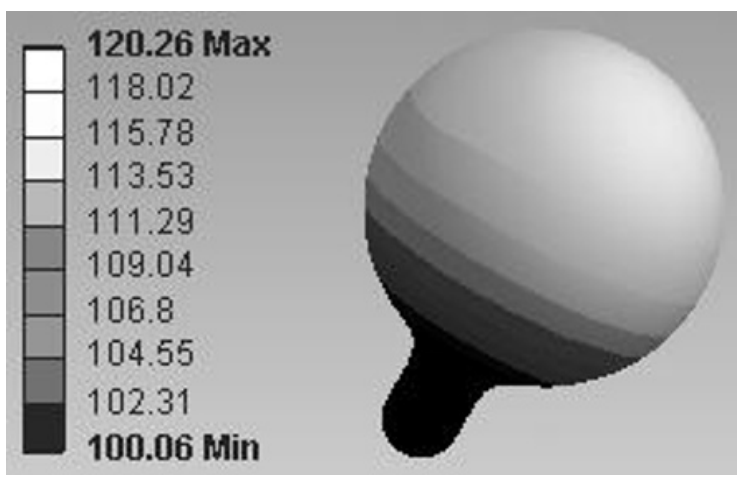

Figure 7. Thermal simulation result of the rubidium bulb in the optimized lamp module.

\section{Experiments}

According to our engineering experience and the operating data of on-board RAFSs, the process of rubidium steady-state distribution in a spectral lamp may be relatively long, such as several months and even more than 1 year, that means a frequency jump may occur in several days or even several months. So it is difficult to carry out frequency jumps observation experiments during the normal operation time of a RAFS. However, the start-up process of the RAFS is the main process for the liquid rubidium to achieve stable redistribution in the bulb, so the start-up process of the RAFS was selected for the experiment of different rubidium spectrum lamps test.

Two rubidium bulbs were selected from the same batch. The two rubidium bulbs have almost identical physical parameters and rubidium quantity, and their test performance was also similar under the same conditions. Then the rubidium bulbs were respectively installed in an original rubidium lamp module and an optimized rubidium lamp module, and the electrical parameters of the components were adjusted appropriately. The two lamps were successively tested in the same physics package. We started the frequency test at 10 minutes after the RAFS was locked, and the test results are shown in figure 8(In order to facilitate comparison of the two curves, the test data was normalized). The frequency test curve of the RAFS with the original designed rubidium lamp turned slowly during start-up, and there was also a slight frequency jump. This indicated that the process of liquid rubidium reaching stable distribution was slow and not smooth enough. On the other hand, the frequency of the RAFS with the optimized rubidium lamp module could quickly reach the stable state after starting up. The experimental result shows that the optimization of the thermal design for rubidium spectrum lamp can effectively promote the rapid and stable distribution of the liquid rubidium in the bulb. That will help the output frequency of the RAFS to reach the normal value quickly and eliminate the frequency jumps of the RAFS.
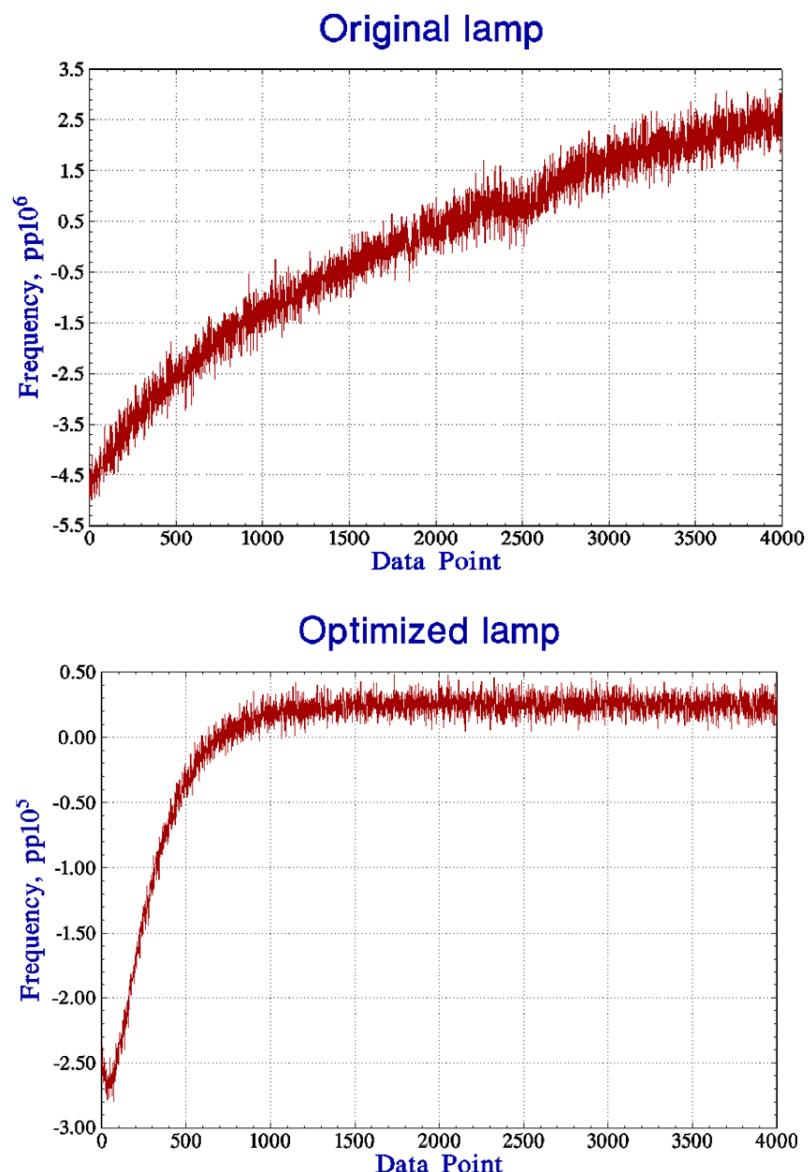

Figure 8. Frequency test of the two lamps during the start-up process.

\section{Conclusions}

The stability of the RAFS is directly related to the positioning accuracy of the navigation system and other satellites. By optimizing the design of rubidium spectral lamp, better temperature gradient was formed in the bulb to make the liquid rubidium in the bulb form a steady state distribution quickly and steadily, which would reduce the rubidium clock frequency jumps and help the output frequency of the RAFS to reach the normal value quickly. The study is benefit to improve the stability of the RAFS. 


\section{References}

1. M. Epstein, 39th Annual Precise Time and Time Interval Meeting (Beverly, MA, USA ,2007):59-78.

2. J. Camparo, IEEE IELCONF(2012):501-508.

3. B.Wang and J.P. Chen, CPGPS 2017 Forum on Cooperative Positioning and Service(Harbin, China,2017):258-261.

4. A. Khare, R. Arora, A. Banik, and S.D. Mehta, IEEE Transactions on Ultrasonics, Ferroelectrics, and Frequency Control, 63, 2, (2016):326-335.

5. J.C. Camparo, 36th Annual Precise Time and Time Interval Meeting (Washington,USA,2004):393-410.
6. J.Z. Cui, T. Yang, L. Zhang, Y.T. Liang, J.H. Tu, W. Yang, J.H. Zhang, Vac \& Cry, 22, 5(2016):271-274.

7. V. Formichella, 32nd URSI GASS (Montreal, Canada, 2017).

8. S.G. He, F. Wang, F Zhao, H.H Wu, G.H. Mei, Acta Metrologica Sinica, 37, 4(2016):433-436.

9. M. Huang, A Stapleton, and J. Comparo, IEEE IFCS2018(Olympic Valley,CA,USA,2018).

10. J.Q. Lian, J.H, Zhang, L.X Wei, 11st China Satellite Navigation Conference(Chengdu,China,2020).(to be published)

11. C. Tao, J.L. Huang, L. Qin, J. Time \& Freq,38, 1(2015) :8-12. 